\title{
Surgical rib fixation as an alternative method of treatment for multiple rib fractures: an audit of results compared with traditional medical management
}

\author{
BI Monzon, ${ }^{1}$ (i) LM Fingleson, ${ }^{2}$ (iD) MS Moeng ${ }^{3}$ (iD \\ ${ }^{1}$ Trauma Unit, Steve Biko Academic Hospital, University of Pretoria, South Africa \\ ${ }^{2}$ Sunninghill Hospital Acute Care and Major Injuries Unit, South Africa \\ ${ }^{3}$ Trauma Unit, Charlotte Maxeke Johannesburg Academic Hospital, University of the Witwatersrand, South Africa
}

Corresponding author, email: bignaciomonzon@gmail.com

Background: Rib fractures are a common cause of morbidity and chronic pain, delaying return to normal activities. Reports suggest that surgical fixation improves acute and long-term outcomes.

Method: A single centre retrospective review of multiple rib fractures, comparing the outcomes of cases managed using surgical fixation with cases managed only with best medical therapy (BMT) over 2 years.

Results: Thirty-five patients with rib fractures were admitted over the study period. The most common causes of rib fractures were motorcycle crashes $(34.2 \%)$ and falls $(31.4 \%)$. Fourteen patients had surgery. There were no differences between the two groups regarding the number of fractured ribs, injury severity score (ISS), ICU or hospital length of stay. The median numeric pain visual analogue scale (VAS) on admission was eight points for non-ventilated patients. In the surgical group the median VAS significantly fell to a median of 2 points in the first 24 hours after surgery $(p=0.04)$. Only two out of 25 major complications were directly attributable to the surgery for rib fixation. Patients managed without surgery needed significantly longer time to return to normal activities compared to those who had surgery (median 7 weeks versus 3 weeks, $p=0.03$ ).

Conclusions: Our preliminary results suggest that rib fixation should be considered a treatment alternative in patients with multiple rib fractures.

Keywords: rib fractures, surgical fixation, flail chest, trauma

\section{Introduction}

Rib fractures are common and causations are multifactorial; they are a recognised marker for severity of injury and a significant cause of in-hospital morbidity, chronic pain, and delays in return to normal activities. ${ }^{1-3}$ The number of ribs fractured, presence of flail segment, patient age, associated lung trauma and extra thoracic injuries, especially traumatic brain injury are predictors of outcome. ${ }^{1-10}$

The current standard of care for rib fractures is nonoperative and is based on several key components. These include appropriate oxygenation, management of respiratory failure with mechanical ventilation, lung re-expansion techniques, appropriate management of pain, removal of secretions and aggressive chest physiotherapy. ${ }^{1-10}$

Unfortunately, non-operative management addresses only the pathophysiological component of this problem, while the mechanical and anatomical problems (actual fractures) are usually overlooked and treated with options that are not designed to facilitate bone consolidation.

The impact of rib fractures on prolonged disability is usually greater than traditionally expected; chronic pain is considered to be present in $22 \%$ of cases (could be as high as
$59 \%$ ) and some form of disability in $53 \%$ of cases 6 months after injury. ${ }^{10-22}$

Attempts to provide rigidity to the chest wall in the event of fractures is not a new proposition, multiple techniques have been used along the years to achieve stability with variable success.

Efforts to advance the surgical fixation of flail chest and multiple rib fractures to the level of standard of care have not met the expectations or received the approval of many surgeons, mostly due to lack of appropriate evidence and familiarity with the procedures. ${ }^{11}$

Evidence in favour of surgical stabilisation of rib fractures has been limited by the quality of the studies as with other trauma related issues, ${ }^{11-20}$ however, the accumulated evidence both from randomised clinical trials and from the systematic reviews and metanalysis consistently favours rib fixation over medical management. ${ }^{1,12-26}$

This mounting experience points to significant advantages such as the reduced incidence of pneumonia and respiratory failure, shorter ventilation time, shorter ICU and hospital stay with minimal complications as well as faster return to productive life, all of which result in improved quality adjusted life-years and costs. ${ }^{1,12-26}$ 
At our institution, all patients with rib fractures were admitted to a trauma ICU ward and offered the standard of care as per protocol of the unit, including intravenous analgesia; oxygen, nebulised bronchodilators as needed, mechanical ventilation when indicated, management of associated injuries and active physiotherapy. A computed tomography scan (CT) of the chest was obtained and three-dimensional (3-D) volume reconstructions performed to evaluate the thoracic skeleton and assess indication for surgery.

As per unit protocol, a follow-up visit was scheduled at two weeks interval after discharge in all cases to clinically assess pain level, physical and pulmonary functionality and obtain chest radiographies to exclude residual pulmonary problems and in the surgical cases to evaluate the fracture site and implant complications. Once the patients re-incorporated to normal activities, they were considered discharged and advised to return for consultation if a problem or concern arose.

Only patients who have three or more rib fractures, or a flail chest with severe pain, as assessed by numeric visual analogue scale (VAS) higher than 6 points, fracture displacement, pulmonary contusion or inability to tolerate physiotherapy are considered for fixation at our institution.

The aim of study was to compare outcomes between subjects with multiple ribs fractures who received surgical rib fixation, and those who only received best medical therapy (BMT).

\section{Method}

Retrospective study from 1 July 2015 to 31 August 2017 (a 25-month period).

\section{Inclusion criteria}

All trauma patients presenting with multiple fractured ribs at a level 2 private trauma centre in Johannesburg.

\section{Exclusion criteria}

Subjects $<18$ years of age, incomplete clinical data, associated severe traumatic brain injury (GCS $<8$ ), major spinal injuries, those with predominantly posterior fractures not amenable to surgical options, and those who have mild symptoms and are able to participate fully in physiotherapy care.

\section{Data collected}

The following information was extracted from clinical notes and entered on a Microsoft Excel Spreadsheet (Microsoft Corp, Redmond, WA): Demographic data (age and sex), mechanism of injury, patterns and number of rib fractures, associated injuries noted, physiological factors (revised trauma score, injury severity score, new injury severity score, probability of survival), medical treatment offered, surgical treatment offered, time to surgery, ICU length of stay, hospital length of stay, in-hospital mortality, procedure related complications, time to return to work or normal activities, pain assessment on admission, after surgery and during outpatient review, and radiological findings during outpatient review.

The surgical procedure to fix the ribs was performed using a muscle sparing thoracic incision (Figure 1). The ribs were stabilised using titanium plates and screws (RibFix Blu $^{\mathrm{TM}}$, Zimmer Biomet, Jacksonville, USA); an effort was always made to provide stability for all accessible fractures.

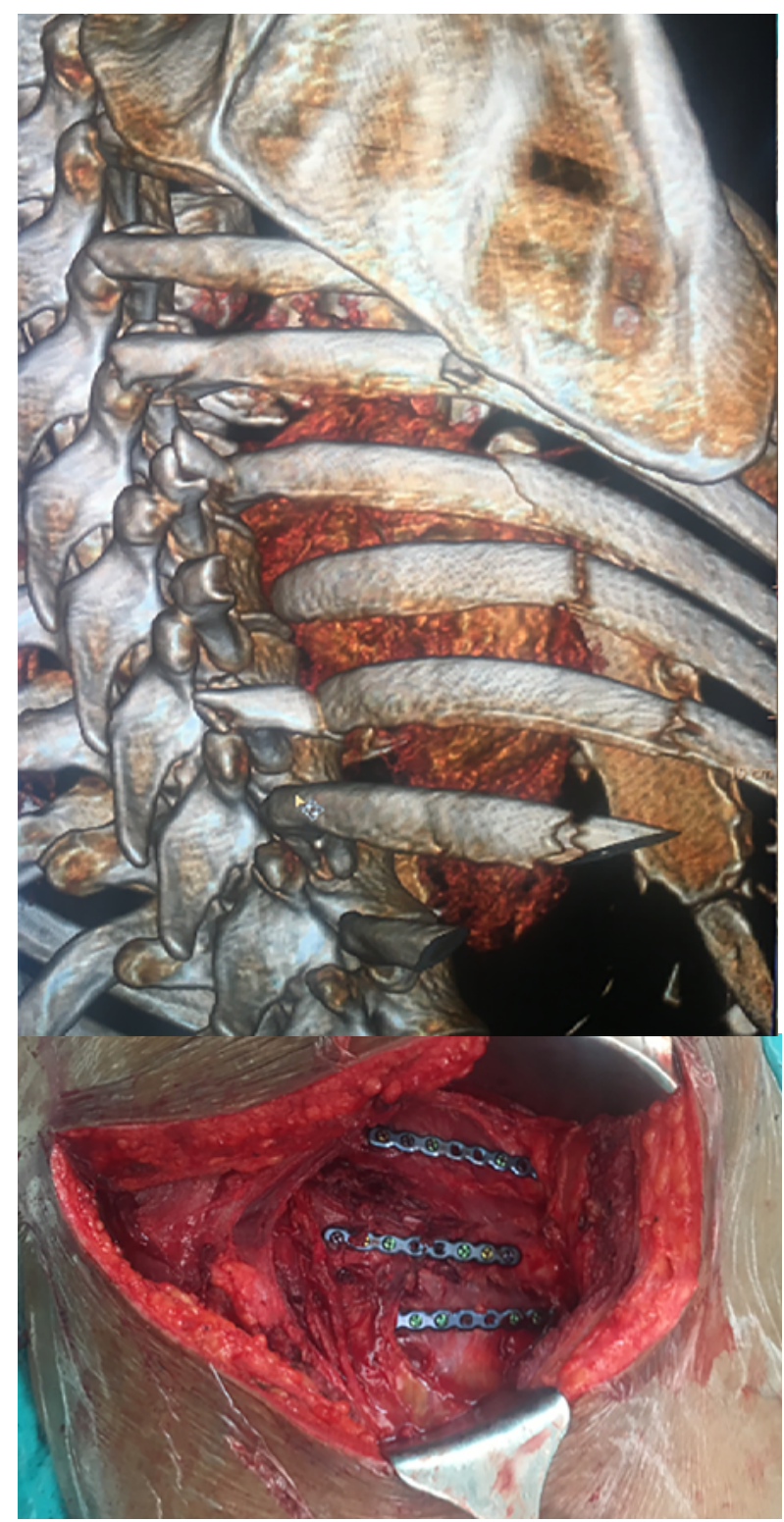

Figure 1: A typical example of multiple rib fractures and three plated fractures

Fractures in ribs one, two, three, ten, eleven and twelve or fractures less than $3 \mathrm{~cm}$ from the costo-vertebral or sternocostal joints were not fixed. The flail segments were either bridged with a long plate spanning the two fractures or using two individual plates.

\section{Data analysis}

Descriptive statistics were used to summarise the data. Statistical difference between comparable groups was assessed using Student's t-test for continuous variables, $p<0.05$ was considered to be statistically significant.

\section{Results}

During the study period, 35 patients were admitted with a diagnosis of rib fractures alone or as part of polytrauma. The patients were mostly males with a median age of 44 years (range 16-68) (Table I).

The most common causes for rib fractures in our series were motorcycle crashes (34.2\%) and falls (31.4\%), closely followed by motor vehicle collisions. More than 
Table I: Demographic information

\begin{tabular}{|c|c|c|c|}
\hline Gender & \multicolumn{2}{|c|}{ Surgery } & Best medical \\
\hline Females & \multicolumn{2}{|c|}{4} & 7 \\
\hline Males & \multicolumn{2}{|c|}{10} & 14 \\
\hline Median age (Range) & \multicolumn{2}{|c|}{43.5 years $(34-68)$} & 45 years $(16-68)$ \\
\hline \multicolumn{4}{|c|}{ Table II: Injury severity score (ISS) } \\
\hline $\begin{array}{l}\text { Severity of injury } \\
\text { (ISS) }\end{array}$ & All & Surgery & Best medical \\
\hline Moderate (1-15) & 2 & & 2 \\
\hline Severe (16-25) & 21 & 10 & 11 \\
\hline Very severe $(26-40)$ & 5 & 1 & 4 \\
\hline Critical (41-75) & 7 & 3 & 4 \\
\hline Median (Range) & $\begin{array}{c}21 \\
(4-75)\end{array}$ & $\begin{array}{c}21 \\
(16-75)\end{array}$ & $\begin{array}{c}24 \\
(4-75)\end{array}$ \\
\hline
\end{tabular}

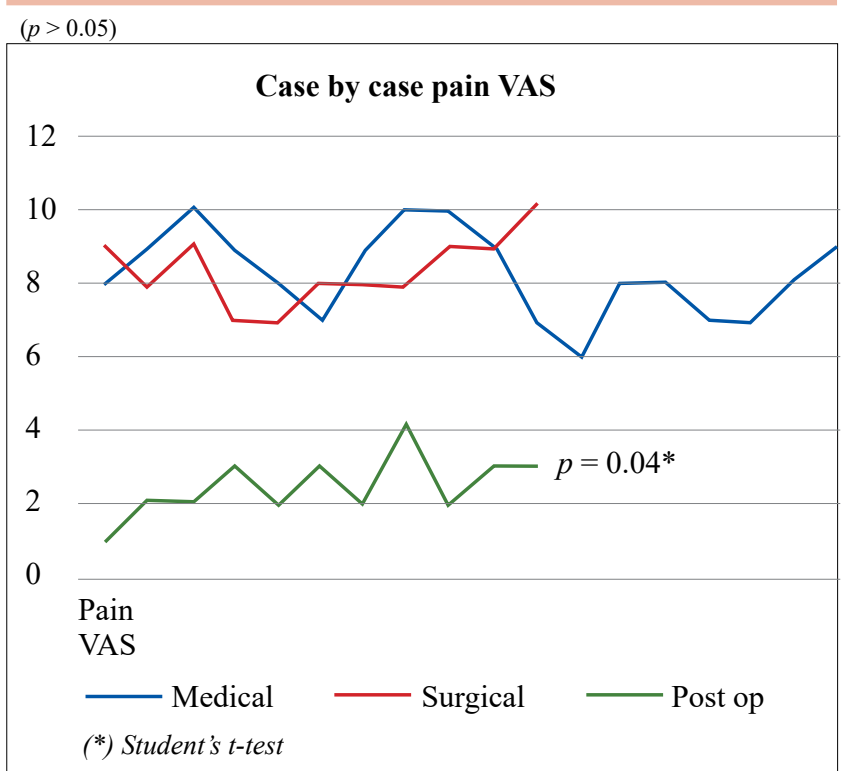

Figure 2: Pain: numeric visual analogue scales (VAS)

sixty per cent of the cases were considered severe trauma (ISS > 16), the median ISS was 21 (4-75). For the patients treated with BMT, the median ISS was 24 (4-75) versus ISS 21 (16-75) for those offered surgery (Table 2).

There was no major difference regarding affected side (15 right - 19 left), and only one patient had bilateral fractures.

Seventy-seven per cent $(77.1 \%)$ of the patients had three or more fractures (median 6; range 3-7) including nine flail chests $(25.7 \%)$. The most common associated injuries were pulmonary contusion in 27 cases $(77.1 \%)$, followed by haemopneumothorax in 24 cases $(68.5 \%)$. Other associated injuries included clavicular fracture, upper and lower limb fractures, ruptured spleen and mild TBI, among others.

Numeric visual pain scale (VAS) assessments were administered to all conscious patients; overall the median VAS pain on admission for non-ventilated patients was 8 points (range 6-10), operated patients who were not ventilated demonstrated significant immediate postoperative reduction of the pain scales to a median of 2 out of 10 (range 1-4) (Figure 2).

Twelve of the fourteen patients $(85.7 \%)$ had the surgery performed in the first seven days following injury (median 4 days, range 2-14).
Table III: Complications (*)

\begin{tabular}{lcc}
\hline & Surgery & Best medical \\
\hline Pneumonia & 4 & 4 \\
Cardiovascular failure & 1 & 2 \\
Severe sepsis & 2 & 1 \\
Acute kidney injury (RRT) & 1 & 2 \\
Retained haemothorax & 1 & 1 \\
Chest wound seroma/hematoma & 2 & - \\
Other & 2 & 2 \\
Total & 13 & 12
\end{tabular}

(*) Some patients had more than one complication recorded RRT - renal replacement therapy

Table IV: Time to return to normal activities

\begin{tabular}{lcc}
\hline Weeks & Surgery & Best medical \\
\hline 3 or less & 8 & \\
4 to 6 & 3 & 10 \\
7 or more & 3 & 11 \\
Average & 5.3 & 9.3 \\
Median (Range) & $3(2-18)^{*}$ & $7(4-36)$ \\
$\left({ }^{*}\right)$ Student's t-test $p=0.003$ & &
\end{tabular}

Twenty-five major complications were recorded, 13 in the surgery group and 12 in the BMT group. The most common complication was pneumonia in $23 \%$ of cases (4 in each group); other complications included cardiovascular failure, severe sepsis and acute kidney injury requiring renal replacement therapy. Complications related to the ORIF procedure were recorded in only 2 of 14 patients operated (Table 3).

ICU and hospital stay were similar for patients operated or managed with BMT (6.5 and 8.5 days, respectively). The median time to return to normal productive life was 3 weeks for the surgical patients (ranging between 2-16 weeks) versus 7 weeks for the BMT group (range 3-52 weeks) (Table 4).

\section{Discussion}

The idea of stabilising fractured ribs to reduce pain, complications and facilitate healing is not new. In 1926, Jones ${ }^{3}$ first described the application of traction to the sternum to treat flail chest. Others soon followed with a myriad of different methods including traction, direct wiring of ribs and metal implants, unfortunately, surgical stabilisation did not become the standard of care, as mechanical ventilation was considered satisfactory for the treatment of the associated pulmonary contusion and to provide stability to the chest wall. $3,5,6$

Over the years, multiple publications have demonstrated a clear reduction in the need for opioid analgesia, incidence of pneumonia, shorter ventilation times and ICU stay and generally, a better outcome when surgery was offered over BMT. 1,2,5,6,10,12-30 Unfortunately, in South Africa, not all patients have access to all of the BMT strategies, including options in advanced pain management.

Our series showed similar results between the two groups regarding ISS, number of fractures, pain VAS on admission, ICU and hospital length of stay and complications; the 
main difference observed was the time necessary to return to normal activities (median 7 weeks for medical therapy versus 3 weeks for surgical cases $[p=0.03]$ ) and an obvious reduction of pain VAS in the postoperative period $(p=0.04)$.

Despite the clear advantage of mechanical ventilation to treat the respiratory failure associated with multiple rib fractures, the use of positive end-expiratory pressure (PEEP) does not achieve complete stability of the thoracic skeleton, which impacts the consolidation of the fracture site. Adequate analgesia, chest physiotherapy and early skeletal (rib) fixation seems to prevent complications, as stated in several recent studies. ${ }^{1,2,13,15-17,19,21}$

\section{Study limitations}

A single centre study with a small sample size having potential selection bias and lack of power. The retrospective nature opens it up to the usual limitations of such studies. The pain VAS is subjective and results may not be accurate.

\section{Conclusion}

There were no significant immediate differences between the ICU length of stay and complications in the BMT group and the surgical group. The postoperative pain VAS was significantly reduced in the surgical group. There was also a significant trend to earlier return to normal activities in the surgical group.

Based on the results of our study and the available evidence, we conclude that rib fixation should be offered to all patients that fit the criteria for surgery.

\section{Conflict of interest}

The authors declare no conflict of interest.

\section{Funding source}

None.

\section{Ethical approval}

Ethical approval was obtained from the University of the Witwatersrand Human Research Ethics Committee M180559.

\section{ORCID}

BI Monzon (iD https://orcid.org/0000-0002-3543-6352 LM Fingleson (iD https://orcid.org/0000-0002-9474-3520 MS Moeng (iD https://orcid.org/0000-0001-7459-3388

\section{REFERENCES}

1. Witt CE, Bulger EM. Comprehensive approach to the management of the patient with multiple rib fractures: a review and introduction of a bundled rib fracture management protocol. Trauma Surg Acute Care Open. 2017;2:e000064. https://doi.org/10.1136/tsaco-2016-000064.

2. Kocher GJ, Al-Hourani M, Minervini F. Surgical fixation of rib fractures: how I do it. J Vis Surg. 2020;6:16. https://doi. org/10.21037/jovs.2019/11/04.

3. Ziegler DW, Agarwal NN. The morbidity and mortality of rib fractures. J Trauma 1994;37(6):975-9.

4. Flagel BT, Luchette FA, Reed RL, et al. Half-a-dozen ribs: the break point for mortality. Surgery. 2005;138:717-23.

5. Tanaka H, Yukioka T, Yamaguti Y, et al. Surgical stabilisation or internal pneumatic stabilisation? A prospective randomised study of management of severe flail chest patients. J Trauma. 2002;52(4):727-32.

6. Dehghan N, De Mestral C, McKee MD, Schemitsch EH, Nathens A. Flail chest injuries: a review of outcomes and treatment practices from National Trauma Data Bank. J Trauma Acute Care Surg. 2014;76:462-8.

7. Bulger E, Arneson MA, Mock CN, Jurkovic GJ. Rib fractures in the elderly. J Trauma. 2000;48(6):1040-6; discussion 10467.

8. Fabricant L, Ham B, Mullins R, Mayberry J. Prolonged pain and disability are common after rib fractures. Am J Surg. 2013;205(5):511-5.

9. Gordy S, Fabricant L, Ham B, Mullins R, Mayberry J. The contribution of rib fractures to chronic pain and disability. Am J Surg. 2014:207(5):659-62; discussion in 662-3.

10. Harrington DT, Philips B, Machan J, et al. Factors associated with survival following blunt chest trauma in older patients: results from a large regional trauma cooperative. Arch Surg. 2010;145(5):432-7.

11. Pressley CM, Fry WR, Philp AS, Berry SD, Smith RS Predicting outcome of patients with chest wall injury. Am J Surg. 2012;204(6):910-3.

12. Leinicke JA, Elmore L, Freeman BD, Colditz GA. Operative management of rib fractures in the setting of flail chest: a systematic review and meta-analysis. Ann Surg. 2013;258(6). https://doi.org/10.1097/SLA.0b013e3182895bb.

13. Granetzny A, El-Aal MA, Emam E, Shalaby A, Boseila A. Surgical versus conservative treatment of flail chest. evaluation of the pulmonary status. Interac Cardiovasc Thorac Surg. 2005;4:583-7. https://doi.org/10.1510/icvts.2005.111807.

14. Richardson JD, Franklin GA, Heffley S, Seligson D. Operative fixation of chest wall fractures: an underused procedure? Am Surg. 2007;73:591-7.

15. Marasco SF, Davies AR, Cooper J, et al. Prospective randomised controlled trial of operative rib fixation in traumatic flail chest. J Am Coll Surg. 2013;216(5):924-32. https://doi.org/.1016/jamcollsurg.2012.12.024.

16. Cataneo AJM, Cataneo DC, De Oliveira FHS, et al. Surgical versus nonsurgical interventions for flail chest. Cochrane Database Syst Rev. 2015;29(7):CD009919. https://doi. org/10.1002/14651858.CD009919.pub2.

17. Cheng MJ. Surgical stabilisation of traumatic flail chest is superior to conservative management. Clin Res Prac. 2019;5(2):eP1948. https://doi.org/10.22237/crp/1568851320.

18. Slobogean GP, MacPherson CA, Pelletier A, Hameed SM. Surgical fixation vs nonoperative management of flail chest: a meta-analysis. J Am Coll Surg. 2013;216:302-11.

19. Swart E, Laratta J, Slobogean G, Mehta S. Operative treatment of rib fractures in flail chest injuries: a meta-analysis and cost effectiveness analysis. J Orthop Trauma. 2017;31(2):64-70(7). https://doi.org/10.1097/BOT.0000000000000750.

20. Pieracci FM, Lin Y, Rodil M, Snyder M. A prospective, controlled clinical evaluation of surgical stabilisation of severe rib fractures. J Trauma Acute Care Surg. 2016;80(2):187-94.

21. Katsoakis G, Hasenboehler EA, Strib EW, et al. Open reduction and internal fixation of rib fractures. Eastern Association for the Surgery of Trauma Practice Management Guideline. J Trauma. 2017;82(3):618-26.

22. Schulte K, Whitaker D, Attia R. In patients with acute flail chest does surgical rib fixation improve outcomes in terms of morbidity and mortality? Interact Cardiovasc Thorac Surg. 2016;23(2):314-9. 
23. De Jong MB, Kokke MC, Hietbrink F, Leenen LP. Surgical management of rib fractures: strategies and literature review. Scand J Surg 2014;103(2):120-25

24. De Moya M, Nirula R, Biffl W. Rib fixation: who, what, when? Trauma Surg Acute Care Open. 2017;2:1-4. https://doi. org/10.1136/tsaco-2016-000059.

25. Pettiford BL, Luketich JD, Landreneau RJ. The management of flail chest. Thorac Surg Clin. 2007;17(1):25-33.

26. Doben AR, Eriksson EA, Denlinger CE, et al. Surgical rib fixation for flail chest deformity improves liberation from mechanical ventilation. J Crit Care. 2014;29(1):139-43.

27. Caragounis EC, Olsen MF, Pazooki D, Granhed H. Surgical treatment of multiple rib fractures and flail chest in trauma: a one-year follow-up study. World J Emerg Surg. 2016;11:2733 .

28. Billé A, Okiror L, Campbell A, Simons J, Routledge T. Evaluation of long-term results and quality of life in patients who underwent rib fixation with titanium devices after trauma. Gen Thorac Cardiovasc Surg. 2013;61(6):345-9.

29. Girsowicz E, Falcoz PE, Santelmo N, Massard G. Does surgical stabilisation improve outcomes in patients with isolated multiple distracted and painful non-flail rib fractures? Internac Cardiovasc Thorac Surg. 2012;14(3):312-5.

30. Marasco S, Cooper J, Pick A, Kossmann T. Pilot study of operative fixation of fractured ribs in patients with flail chest. ANZ J Surg. 2009;79(11):804-8. 